OPEN ACCESS

Edited by:

Rebeca Martín

INRA Centre Jouy-en-Josas, France

Reviewed by:

Muriel Derrien

Danone, France

Nuria Salazar,

Instituto de Productos Lácteos de Asturias (IPLA-CSIC), Spain

*Correspondence:

Patrice D. Can

patrice.cani@uclouvain.be

Specialty section:

This article was submitted to

Food Microbiology,

a section of the journal

Frontiers in Microbiology

Received: 03 July 2017

Accepted: 31 August 2017

Published: 22 September 2017

Citation:

Cani PD and de Vos WM (2017) Next-Generation Beneficial Microbes: The Case of Akkermansia muciniphila.

Front. Microbiol. 8:1765

doi: 10.3389/fmicb.2017.01765

\section{Next-Generation Beneficial Microbes: The Case of Akkermansia muciniphila}

\author{
Patrice D. Cani ${ }^{1 *}$ and Willem M. de $\operatorname{Vos}^{2,3}$ \\ ${ }^{1}$ Walloon Excellence in Life Sciences and Biotechnology (WELBIO), Metabolism and Nutrition Research Group, Louvain \\ Drug Research Institute, Université catholique de Louvain, Brussels, Belgium, ${ }^{2}$ Laboratory of Microbiology, Wageningen \\ University, Wageningen, Netherlands, ${ }^{3}$ Immunobiology Research Program, Research Programs Unit, Department of \\ Bacteriology and Immunology, University of Helsinki, Helsinki, Finland
}

Metabolic disorders associated with obesity and cardiometabolic disorders are worldwide epidemic. Among the different environmental factors, the gut microbiota is now considered as a key player interfering with energy metabolism and host susceptibility to several non-communicable diseases. Among the next-generation beneficial microbes that have been identified, Akkermansia muciniphila is a promising candidate. Indeed, A. muciniphila is inversely associated with obesity, diabetes, cardiometabolic diseases and low-grade inflammation. Besides the numerous correlations observed, a large body of evidence has demonstrated the causal beneficial impact of this bacterium in a variety of preclinical models. Translating these exciting observations to human would be the next logic step and it now appears that several obstacles that would prevent the use of $A$. muciniphila administration in humans have been overcome. Moreover, several lines of evidence indicate that pasteurization of A. muciniphila not only increases its stability but more importantly increases its efficacy. This strongly positions $A$. muciniphila in the forefront of next-generation candidates for developing novel food or pharma supplements with beneficial effects. Finally, a specific protein present on the outer membrane of $A$. muciniphila, termed Amuc_1100, could be strong candidate for future drug development. In conclusion, as plants and its related knowledge, known as pharmacognosy, have been the source for designing drugs over the last century, we propose that microbes and microbiomegnosy, or knowledge of our gut microbiome, can become a novel source of future therapies.

Keywords: Akkermansia muciniphila, obesity, diabetes mellitus, type 2, probiotics and prebiotics, gut barrier function

\section{INTRODUCTION}

Overweight and obesity have reached epidemic proportions with more than 600 million of adults and 100 million children of the world's population suffering from obesity (GBD 2015 Obesity Collaborators et al., 2017). Obesity predisposes to the development of type 2 diabetes and cardiovascular diseases. These two pathologies are part of the metabolic syndrome that is also becoming major problem in public health (Abdelaal et al., 2017; Ajala et al., 2017). Gut microbes play an important role in the regulation of host metabolism and low-grade inflammation (Hartstra et al., 2015; Marchesi et al., 2016; Cani, 2017). The perturbation of the composition and the 
activity of the gut microbiota, also known as dysbiosis, is thought to be involved in the emergence of the metabolic syndrome (Wen and Duffy, 2017). Nowadays, numerous studies have demonstrated that our dietary habits strongly influence the composition and function of the gut microbiota and eventually may contribute to the onset or the protection against metabolic disorders (David et al., 2014; Korpela et al., 2014; Salonen et al., 2014; Carmody et al., 2015; Zeevi et al., 2015; Cani and Everard, 2016; Thaiss et al., 2016).

Well documented among the potential ways to affect the gut microbiota, is the consumption of selected microbes that are marketed as probiotics defined as "live microorganisms that when administered in adequate amounts confer a health benefit on the host" (Hill et al., 2014). It is worth noting that the current majority of probiotics sold on the market include mainly microorganisms from the genera Lactobacillus and Bifidobacterium (Douillard and de Vos, 2014). However, other ways such as the consumption of prebiotics have gained considerable attention over the last 20 years (Roberfroid et al., 2010). The prebiotic concept, discovered in Gibson and Roberfroid (1995), has led to a great number of dietary supplements that is an important growth market. The definition of prebiotic is now widely used and has been recently revised as "a substrate that is selectively utilized by host microorganisms conferring a health benefit" (Gibson et al., 2017). Thus, nutritional components that escape the digestion in the upper alimentary tract may have an impact on the gut microbiota by modulating some members of the gut microbiota its composition and its activity. However, the concept of prebiotic has not yet revealed all its secrets. In spite of numerous discoveries of molecular mechanisms explaining how prebiotics and the gut microbiota interact with the host, it remains difficult to identify the bacterial candidate(s) involved in the beneficial effects observed on the energy, glucose, lipid metabolism and immunity.

\section{FROM PREBIOTIC TO NEXT-GENERATION BENEFICIAL MICROBE: FOCUS ON THE IDENTIFICATION OF Akkermansia muciniphila}

Akkermansia muciniphila is one of the most abundant single species in the human intestinal microbiota $(0.5-5 \%$ of the total bacteria) and has been isolated and characterized as a mucinutilizing specialist in 2004 by Muriel Derrien in her Ph.D. research at Wageningen University (Derrien et al., 2004; Collado et al., 2007). This discovery was initiated by the notion that the human body produces its own "prebiotics" or microbial substrates, namely mucus, an abundant glycoprotein that is specifically produced and degraded in the colon (Ouwehand et al., 2005). While germ-free mouse experiments showed that A. muciniphila showed immune and metabolic signaling, specifically in the colon, the exact functions of this unusual microbe remained enigmatic (Derrien et al., 2008, 2011).
Further indications for the function of A. muciniphila were subsequently determined in other prebiotic studies using inulintype fructans that were initially characterized as bifidogenic compounds able to increase the abundance of Bifidobacterium spp. (Gibson and Roberfroid, 1995). Thanks to the development of novel culture-independent techniques, we decided to revise in depth the impact of such kind of prebiotics on the overall microbial community in mice. Therefore, in search of potential novel bacterial candidates, we combined different techniques (phylogenetic microarray, high-throughput sequencing, gradient denaturation gel and qPCR), which allowed us to analyze and to compare all the bacteria that were present in the intestinal microbiota. The first surprise was to discover that more than 100 different taxa were affected by prebiotics (Everard et al., 2011; Everard et al., 2014). Among these bacteria, we found that the relative abundance of $A$. muciniphila increased more than 100 -fold following the ingestion of prebiotics thereby reaching the abundance of up to $4.5 \%$ under high-fat diet (Everard et al., 2014), whereas this effect was lower under normal chow diet (0.09-2.5\%) depending on the model (Everard et al., 2011, 2014). It is worth noting that these findings are confirmed in different set of experiments (Everard et al., 2013; Liu et al., 2016; Reid et al., 2016; Catry et al., 2017; Zhu et al., 2017). Interestingly, we and others discovered that A. muciniphila was less abundant in the intestinal microbiota of both genetic and diet-induced obese and diabetic mice (Everard et al., 2011, 2013, 2014; Schneeberger et al., 2015; Leal-Diaz et al., 2016; Ojo et al., 2016; Song et al., 2016; Singh et al., 2017), however, few studies reported in mice an increased abundance of A. muciniphila upon the ingestion of a high-fat high sucrose diet (Anhe et al., 2015; Carmody et al., 2015). It has also been largely demonstrated that inulintype fructans feeding improves metabolic disorders associated with obesity, including a decreased fat mass, insulin resistance, lower liver steatosis and a reinforcement of the gut barrier (Figure 1) (Cani et al., 2004, 2006, 2009; Maurer et al., 2010; Everard et al., 2011; Pachikian et al., 2012; Greer et al., 2016). Importantly, in humans the abundance of $A$. muciniphila was decreased in several pathological situations such as obesity, type 2 diabetes, inflammatory bowel diseases, hypertension and liver diseases (Png et al., 2010; Belzer and de Vos, 2012; Zhang et al., 2013; Dao et al., 2015; Yassour et al., 2016; Grander et al., 2017; Li et al., 2017). Conversely, antidiabetic treatments, such as metformin administration and bariatric surgery were both found to be associated with a marked increase in the abundance of A. muciniphila (Figure 1) (Shin et al., 2014; Forslund et al., 2015; de la Cuesta-Zuluaga et al., 2017). Therefore, a large body of evidence suggested that A. muciniphila may contribute to protect from specific metabolic disorders and cardiometabolic risk factors associated with a low-grade inflammatory tone.

\section{ADMINISTRATION OF Akkermansia muciniphila: MULTIPLE EFFECTS ON THE GUT AND BEYOND}

Inspired by the numerous indications that the relative levels of A. muciniphila decreased during obesity and metabolic disorders 


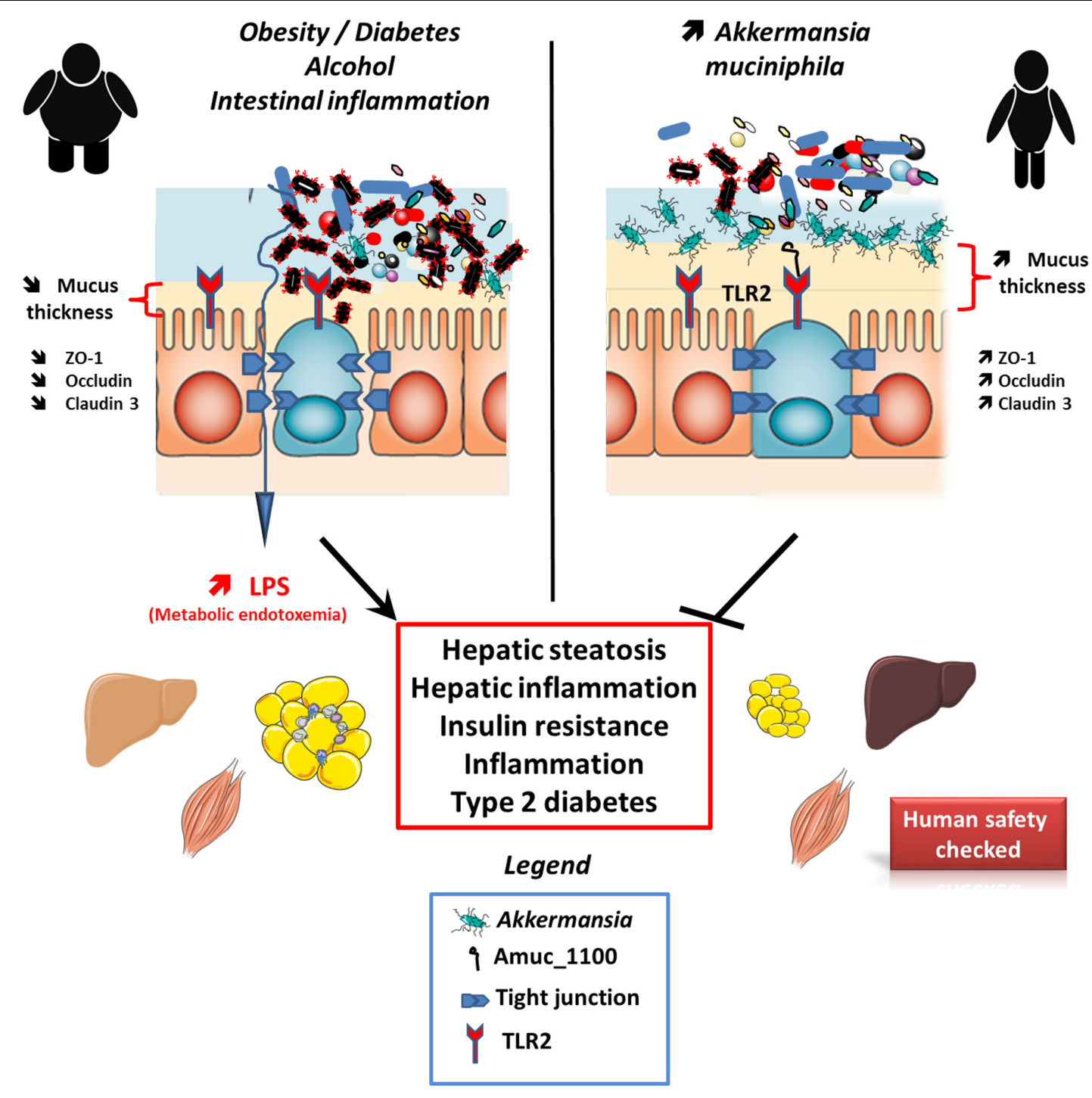

FIGURE 1 | Effects of $A$. muciniphila and derived products on host metabolism. A. muciniphila has been found to be lower in several conditions such as during obesity, diabetes, intestinal inflammation, liver diseases, or chronic alcohol consumption. This is associated with an altered gut barrier function leading to an increased plasma LPS levels and eventually triggering low grade inflammation and metabolic disorders. A. muciniphila alive or pasteurized as well as Amuc_1100 has been shown to restore gut barrier function likely by acting on TLR2 and restoring appropriate tight junction expression. All these results are associated with an increased mucus later thickness and an improvement of metabolic disorders. It is worth noting that an exploratory human investigation has shown that A. muciniphila is apparently safe.

in mouse and man, we decided to study the causal link between A. muciniphila and improvements in metabolism. This was done by investigating the impact of a daily oral supplementation with live A. muciniphila on the onset of obesity, diabetes and gut barrier dysfunction in mice. We found that the administration of live A. muciniphila at the dose of $2.10^{8}$ bacterial cells per day was partly protecting against diet-induced obesity in mice (Everard et al., 2013). Indeed, mice showed a 50\% lower body weight gain when treated with live A. muciniphila without altering neither their dietary food intake nor the elimination of dietary fats in fecal matter. This protection was mirrored by two times less visceral and subcutaneous fat mass (Figure 1), but also by increased markers of fatty acid oxidation in the adipose tissue (Everard et al., 2013). In addition, animals receiving live A. muciniphila did no longer exhibited insulin resistance, nor infiltration of inflammatory cells (CD11c) in the adipose tissue, which is a key characteristic of obesity and associated low-grade inflammation (Everard et al., 2013). Interestingly, most of all the metabolic improvements observed following treatment with live A. muciniphila were in the range as those observed following oligofructose or inulin treatment (Cani et al., 2009; Dewulf et al., 2011; Everard et al., 2011, 2014), although live A. muciniphila was not affecting food intake behavior as do prebiotics like inulin and oligofructose. 
Knowing that these metabolic features can be caused by an increased plasma LPS level (i.e., metabolic endotoxemia) or bacterial translocation (Cani et al., 2007; Amar et al., 2011), we next investigated the gut barrier function by measuring several markers. We observed that live A. muciniphila prevented the development of metabolic endotoxemia, an effect associated with the restoration of a normal mucus layer thickness (Figure 1) (Everard et al., 2013). We also found that administration of live A. muciniphila restored the endogenous production of antimicrobial peptides. We then discovered that live A. muciniphila increased the endogenous production of specific bioactive lipids that belongs to the endocannabinoid family and are known to have anti-inflammatory activities and regulating the endogenous production of gut peptides involved in glucose regulation and gut barrier, respectively, glucagon-like peptide-1 and 2 (GLP-1 and GLP-2) (Cani et al., 2016). It is worth noting that all these findings have subsequently been confirmed by different groups and extended to other specific disorders such as atherosclerosis, hepatic inflammation and hypercholesterolemia (Shin et al., 2014; Li et al., 2016; Shen et al., 2016; Grander et al., 2017; Plovier et al., 2017).

Collectively all these data reinforce the assumption that live A. muciniphila can be considered as a next-generation beneficial microbe with the exceptional particularity that this bacterium can act on numerous facets of the metabolic syndrome and cardiometabolic disorders. Still, these discoveries have raised different fundamental questions that will still have to be studied in humans with the aim to generate new therapeutic tools.

\section{CROSSING THE BARRIER OF SPECIES: FROM MICE TO MAN}

Akkermansia muciniphila requires specific culture conditions and complex animal-based medium (i.e., mucin from animal source) and although it may respire under microaerophilic conditions, the cells are relatively sensitive to oxygen (Ouwerkerk et al., 2016). These properties complicate the administration of $A$. muciniphila to human as to evaluate its potential, hence limiting its therapeutic perspectives. In order to solve this problem, a synthetic medium was developed in order to allow the culture of A. muciniphila with a high yield and devoid of compounds incompatible with administration in humans (Plovier et al., 2017; Van der Ark et al., unpublished data). Besides the successful development of this synthetic medium, the previous assessment of the efficacy of $A$. muciniphila were performed with cells grown on a mucin-based medium. Therefore, the bacteria cultured on the different media were tested and compared. Interestingly, $A$. muciniphila retains its effectiveness independently of the medium used, and as previously observed, mice treated with the bacterium gained less weight, exhibited an improved glucose tolerance, and insulin resistance under hyperlipidic diet (Figure 1) (Plovier et al., 2017).

\section{SERENDIPITY: THE UNEXPECTED ADVANTAGE OF PASTEURIZATION}

In 2013, it was showed that the protective effects of $A$. muciniphila disappeared when the bacterium was destroyed by using autoclaving, a heat treatment that destroyes all the constituents of bacteria and spores (Everard et al., 2013). As A. muciniphila is a Gram-negative bacterium and hence no spore-former, we were interested what the effects would be of pasteurization, a milder heat inactivation method than autoclaving. Therefore, we tested the impact of administrating pasteurized $A$. muciniphila $\left(30 \mathrm{~min}\right.$ at $70^{\circ} \mathrm{C}$ ) cells on diet-induced metabolic disorders in mice. Unexpectedly, this method of inactivation did not abolish the effect of $A$. muciniphila but even exacerbated its beneficial impact. Specifically, mice receiving the pasteurized bacterium and the high-fat diet had a similar body weight gain and fat mass than those observed in mice fed a control diet. Again, these effects were independent of the food intake but pasteurized A. muciniphila increased the loss of energy in the feces of the treated mice, indicating a decrease in energy absorption that could contribute to explain the lower weight gain. Pasteurized A. muciniphila also strongly improved glucose tolerance, hepatic insulin sensitivity, and completely blocked the diet-induced metabolic endotoxemia. Although, the mechanisms of action of the bacteria are not yet fully elucidated, it is known that A. muciniphila express numerous highly abundant protein on its outer membrane (Ottman et al., 2017). Among these proteins, Amuc_1100, implicated in the formation of pili by A. muciniphila, was one of the most abundant (Plovier et al., 2017).

\section{Akkermansia muciniphila: A GATE KEEPER THAT DIALOGS WITH THE INNATE IMMUNE SYSTEM}

We previously found that A. muciniphila was able to restore the expression of specific antimicrobial peptides (Everard et al., 2013). However, Nucleotide oligomerization domain (NOD)like receptors (NLRs) and Toll-Like Receptors (TLRs) are a specialized group of membrane and intracellular proteins that play a critical role in the regulation of immunity and are directly involved in the recognition of bacterial constituents by the immune system. Therefore, we evaluated the potential of A. muciniphila to activate different NOD and TLRs. Strikingly, we found that the bacteria specifically interact with TLR2. TLR2 has been shown to modulate intestinal homeostasis and host metabolism (Caricilli et al., 2011; Brun et al., 2013), thereby participating in the interactions between microbes and host. In addition, to better characterize the interaction between A. muciniphila and this receptor, we took advantage of genomic and proteomic analyzes of the external membrane of the bacterium, which may be exposed to host receptors (Ottman et al., 2016). Among these proteins, Amuc_1100 was one of the most abundant. This protein is implicated in the formation of pili by $A$. muciniphila and thus could participate in the interaction between the bacterium and TLR2. This hypothesis was further 
confirmed by showing that a version of the genetically engineered protein (called Amuc_1100*) was effectively activating TLR2 and with the same magnitude as A. muciniphila. In addition, Amuc_1100* remained stable at the temperature used during pasteurization, and could therefore contribute to the effects of the pasteurized bacterium. Amuc_1100* was also able to replicate almost all the effects of $A$. muciniphila alive or pasteurized in high-fat diet fed mice. A. muciniphila, whether live or pasteurized, and Amuc_1100* also decreased high cholesterol levels induced by the high-fat diet. Conversely, the pasteurized bacterium specifically also reduced the triglyceridemia of the treated mice, reinforcing the idea that the pasteurization of A. muciniphila reinforces its protective effects. A potential mechanism explaining this could be the exposure of active molecules by the heat treatments, including Amuc_1100, or the inactivation of inhibitory compounds, or combinations thereof.

\section{FIRST ASSESSMENT OF Akkermansia muciniphila IN HUMANS WITH METABOLIC SYNDROME}

As discussed earlier, A. muciniphila has various advantages as compared to other beneficial microbes and specific probiotics, at least in the context of the metabolic syndrome. A. muciniphila is present in the human milk, is highly abundant in lean and nondiabetic subjects, and is even highly increased upon metformin treatment of gastric bypass surgery, and this without obvious deleterious impact. This unique character does not preclude the fact the human investigations and safety assessment must be done. Hence, to become a putative future food supplement, the safety must be tested. We evaluated the toxicity and the emergence of possible side effects related to the administration of A. muciniphila in humans (20 subjects) as part of an ongoing clinical trial of individuals with metabolic syndrome (Plovier et al., 2017). To this end, we analyzed relevant clinical parameters related to liver, muscles and renal functions as well as markers of immunity and inflammation in individuals who received A. muciniphila daily for 2 weeks and then extended to 3 months. Whatever the formulation of A. muciniphila (live at $10^{9}$ and $10^{10}$ bacteria per day or pasteurized at $10^{10}$ bacteria per day), no changes were observed for the markers tested after 2 weeks or 3 months of daily administration. In addition, the frequency of side effects reported by patients were similar in the different groups. These first data indicate that A. muciniphila (active or pasteurized) is tolerated in individuals with metabolic syndrome and is likely not toxic.

While A. muciniphila is one of the handful of core microbes identified in the intestinal microbiota of over 1000 human adults (Shetty et al., 2017), the administration of its cells, either in live or pasteurized form, in a dietary supplement may be subject to regulatory frameworks that aim to safeguard the consumer. The regulatory requirements relating to the use of live $A$. muciniphila have recently been addressed (Gomez-Gallego et al., 2016). This review summarized the recent comprehensive studies related to A. muciniphila and its safety properties and provided criteria be addressed when A. muciniphila cells are to be considered as a novel food by the European Food Safety Authority in Europe. One aspect that is relevant here and applies to other core intestinal microbes as well, is the fact that most if not all healthy subjects carry these anaerobes. So these have to be consumed at some stage and in this context it is important to note that A. muciniphila is present in early life microbiota and has been detected in mothers' milk (Collado et al., 2007, 2012; Derrien et al., 2008; Jeurink et al., 2013; Ward et al., 2013). Another aspect relates to the antibiotic resistance of $A$. muciniphila that has been studied to some extent in healthy human subjects that carried high levels of $A$. muciniphila-like bacteria and apparently were sensitive to penicillin and tetracycline derivatives but resistant to vancomycin (Dubourg et al., 2013). This was confirmed in in vitro studies on the antibiotic resistance profile with the type strain Amuc $^{\mathrm{T}}$ (Ouwerkerk Ph.D. Thesis Wageningen University 2016). Moreover, inspection of the genome sequence did not reveal antibiotic resistance genes that are linked to known genetically transferrable elements (Gomez-Gallego et al., 2016).

\section{CONCLUSION}

Since its discovery in 2004, numerous studies have mostly linked the abundance of $A$. muciniphila with beneficial effects, and this although very few exceptions exist in specific non-physiological models (i.e., gnotobiotic models, specific immune double knockout models) (Seregin et al., 2017).

Nowadays, A. muciniphila is widely considered as a novel potential candidate to improve metabolic disorders associated with obesity, diabetes, liver diseases and cardiometabolic disorders. Indeed, its administration has been shown to profoundly reduce the development of such diseases.

Other important steps toward the development of A. muciniphila as a next-generation beneficial microbe have been successfully reached. First, the discovery that $A$. muciniphila remained effective by being grown on a synthetic medium compatible with administration in humans. Second, the discovery that inactivation of the bacteria by pasteurization improved its effects, and thus its stability and potential shelf life. Third, the identification of a key mechanisms of interaction between A. muciniphila and its host via the identification of Amuc_1100, and last but not least; fourth, the demonstration that A. muciniphila may be safely administered in the human targeted population.

Finally, the pasteurized bacteria and the identification and the isolation of bacterial constituents such as the relatively small 30$\mathrm{kDa}$ Amuc_1100 open the door to putative development of drugs based on $A$. muciniphila-related product that could also target pathologies such as type 1 diabetes, inflammatory bowel diseases or diseases where the intestinal barrier function is compromised.

\section{AUTHOR CONTRIBUTIONS}

PC and WdV: Conceptualized the review content. 


\section{FUNDING}

PC is senior research associate at FRS-FNRS (Fonds de la Recherche Scientifique). PC is recipient of grants from FNRS (Project de Recherche, convention: T.0138.14) and Walloon region DG06-FSO project (Microbes 1510053). This work was supported by the FRFS-WELBIO under grant: WELBIO-CR2012S-02R. This work is supported in part by the Funds Baillet Latour (Grant for Medical Research 2015). PC is a recipient of POC ERC grant 2016 (European Research Council, Microbes4U_713547) and ERC Starting Grant 2013 (Starting grant 336452-ENIGMO). WdV is partially supported

\section{REFERENCES}

Abdelaal, M., le Roux, C. W., and Docherty, N. G. (2017). Morbidity and mortality associated with obesity. Ann. Transl. Med. 5:161. doi: 10.21037/atm.2017. 03.107

Ajala, O., Mold, F., Boughton, C., Cooke, D., and Whyte, M. (2017). Childhood predictors of cardiovascular disease in adulthood. A systematic review and meta-analysis. Obes. Rev. 18, 1061-1070. doi: 10.1111/obr.12561

Amar, J., Chabo, C., Waget, A., Klopp, P., Vachoux, C., Bermudez-Humaran, L. G., et al. (2011). Intestinal mucosal adherence and translocation of commensal bacteria at the early onset of type 2 diabetes: molecular mechanisms and probiotic treatment. EMBO Mol. Med. 3, 559-572. doi: 10.1002/emmm. 201100159

Anhe, F. F., Roy, D., Pilon, G., Dudonne, S., Matamoros, S., Varin, T. V., et al. (2015). A polyphenol-rich cranberry extract protects from diet-induced obesity, insulin resistance and intestinal inflammation in association with increased Akkermansia spp. population in the gut microbiota of mice. Gut 64, 872-883. doi: 10.1136/gutjnl-2014-307142

Belzer, C., and de Vos, W. M. (2012). Microbes inside-from diversity to function: the case of Akkermansia. ISME J. 6, 1449-1458. doi: 10.1038/ismej.2012.6

Brun, P., Giron, M. C., Qesari, M., Porzionato, A., Caputi, V., Zoppellaro, C., et al. (2013). Toll-like receptor 2 regulates intestinal inflammation by controlling integrity of the enteric nervous system. Gastroenterology 145, 1323-1333. doi: 10.1053/j.gastro.2013.08.047

Cani, P. D. (2017). Gut microbiota - at the intersection of everything? Nat. Rev. Gastroenterol. Hepatol. 14, 321-322. doi: 10.1038/nrgastro.2017.54

Cani, P. D., Amar, J., Iglesias, M. A., Poggi, M., Knauf, C., Bastelica, D., et al. (2007). Metabolic endotoxemia initiates obesity and insulin resistance. Diabetes Metab. Res. Rev. 56, 1761-1772. doi: 10.2337/db06-1491

Cani, P. D., Dewever, C., and Delzenne, N. M. (2004). Inulin-type fructans modulate gastrointestinal peptides involved in appetite regulation (glucagonlike peptide-1 and ghrelin) in rats. Br. J. Nutr. 92, 521-526. doi: 10.1079/ BJN20041225

Cani, P. D., and Everard, A. (2016). Talking microbes: when gut bacteria interact with diet and host organs. Mol. Nutr. Food Res. 60, 58-66. doi: 10.1002/mnfr. 201500406

Cani, P. D., Knauf, C., Iglesias, M. A., Drucker, D. J., Delzenne, N. M., and Burcelin, R. (2006). Improvement of glucose tolerance and hepatic insulin sensitivity by oligofructose requires a functional glucagon-like peptide 1 receptor. Diabetes Metab. Res. Rev. 55, 1484-1490. doi: 10.2337/db05-1360

Cani, P. D., Plovier, H., Van Hul, M., Geurts, L., Delzenne, N. M., Druart, C., et al. (2016). Endocannabinoids - at the crossroads between the gut microbiota and host metabolism. Nat. Rev. Endocrinol. 12, 133-143. doi: 10.1038/nrendo. 2015.211

Cani, P. D., Possemiers, S., Van de, W. T., Guiot, Y., Everard, A., Rottier, O., et al. (2009). Changes in gut microbiota control inflammation in obese mice through a mechanism involving GLP-2-driven improvement of gut permeability. Gut 58, 1091-1103. doi: 10.1136/gut.2008.165886

Caricilli, A. M., Picardi, P. K., de Abreu, L. L., Ueno, M., Prada, P. O., Ropelle, E. R., et al. (2011). Gut microbiota is a key modulator of insulin resistance in TLR 2 knockout mice. PLOS Biol. 9:e1001212. doi: 10.1371/journal.pbio.1001212 by ERC Advanced Grant 250172 (Microbes Inside), the SIAM Gravity Grant 024.002.002 and Spinoza Award of the Netherlands Organization for Scientific Research, and Grants 137389, 141140 and 1272870 of the Academy of Finland.

\section{ACKNOWLEDGMENT}

We thank all the collaborators who have contributed to 10 years of Akkermansia discoveries, Dr. M. Derrien, Dr. C. Belzer, Dr. A. Everard, and Dr. H. Plovier.

Carmody, R. N., Gerber, G. K., Luevano, JM Jr, Gatti, D. M., Somes, L., Svenson, K. L., et al. (2015). Diet dominates host genotype in shaping the murine gut microbiota. Cell Host Microbe 17, 72-84. doi: 10.1016/j.chom.2014.11.010

Catry, E., Bindels, L. B., Tailleux, A., Lestavel, S., Neyrinck, A. M., Goossens, J. F., et al. (2017). Targeting the gut microbiota with inulin-type fructans: preclinical demonstration of a novel approach in the management of endothelial dysfunction. Gut doi: 10.1136/gutjnl-2016-313316 [Epub ahead of print].

Collado, M. C., Derrien, M., Isolauri, E., de Vos, W. M., and Salminen, S. (2007). Intestinal integrity and Akkermansia muciniphila, a mucin-degrading member of the intestinal microbiota present in infants, adults, and the elderly. Appl. Environ. Microbiol. 73, 7767-7770. doi: 10.1128/AEM.01477-07

Collado, M. C., Laitinen, K., Salminen, S., and Isolauri, E. (2012). Maternal weight and excessive weight gain during pregnancy modify the immunomodulatory potential of breast milk. Pediatr. Res. 72, 77-85. doi: 10.1038/pr.2012.42

Dao, M. C., Everard, A., Aron-Wisnewsky, J., Sokolovska, N., Prifti, E., Verger, E. O., et al. (2015). Akkermansia muciniphila and improved metabolic health during a dietary intervention in obesity: relationship with gut microbiome richness and ecology. Gut 65, 426-436. doi: 10.1136/gutjnl-2014-308778

David, L. A., Maurice, C. F., Carmody, R. N., Gootenberg, D. B., Button, J. E., Wolfe, B. E., et al. (2014). Diet rapidly and reproducibly alters the human gut microbiome. Nature 505, 559-563. doi: 10.1038/nature12820

de la Cuesta-Zuluaga, J., Mueller, N. T., Corrales-Agudelo, V., Velasquez-Mejia, E. P., Carmona, J. A., Abad, J. M., et al. (2017). Metformin is associated with higher relative abundance of mucin-degrading Akkermansia muciniphila and several short-chain fatty acid-producing microbiota in the gut. Diabetes Care 40, 54-62. doi: 10.2337/dc16-1324

Derrien, M., Collado, M. C., Ben-Amor, K., Salminen, S., and de Vos, W. M. (2008). The Mucin degrader Akkermansia muciniphila is an abundant resident of the human intestinal tract. Appl. Environ. Microbiol. 74, 1646-1648. doi: 10.1128/AEM.01226-07

Derrien, M., Van Baarlen, P., Hooiveld, G., Norin, E., Muller, M., and de Vos, W. M. (2011). Modulation of mucosal immune response, tolerance, and proliferation in mice colonized by the mucin-degrader Akkermansia muciniphila. Front. Microbiol. 2:166. doi: 10.3389/fmicb.2011.00166

Derrien, M., Vaughan, E. E., Plugge, C. M., and de Vos, W. M. (2004). Akkermansia muciniphila gen. nov., sp. nov., a human intestinal mucin-degrading bacterium. Int. J. Syst. Evol. Microbiol. 54(Pt 5), 1469-1476. doi: 10.1099/ijs.0.02873-0

Dewulf, E. M., Cani, P. D., Neyrinck, A. M., Possemiers, S., Holle, A. V., Muccioli, G. G., et al. (2011). Inulin-type fructans with prebiotic properties counteract GPR43 overexpression and PPARgamma-related adipogenesis in the white adipose tissue of high-fat diet-fed mice. J. Nutr. Biochem. 22, 712-722. doi: 10.1016/j.jnutbio.2010.05.009

Douillard, F. P., and de Vos, W. M. (2014). Functional genomics of lactic acid bacteria: from food to health. Microb. Cell Fact. 13(Suppl. 1), S8. doi: 10.1186/ 1475-2859-13-S1-S8

Dubourg, G., Lagier, J. C., Armougom, F., Robert, C., Audoly, G., Papazian, L., et al. (2013). High-level colonisation of the human gut by Verrucomicrobia following broad-spectrum antibiotic treatment. Int. J. Antimicrob. Agents 41, 149-155. doi: 10.1016/j.ijantimicag.2012.10.012

Everard, A., Belzer, C., Geurts, L., Ouwerkerk, J. P., Druart, C., Bindels, L. B., et al. (2013). Cross-talk between Akkermansia muciniphila and intestinal epithelium 
controls diet-induced obesity. Proc. Natl. Acad. Sci. U.S.A. 110, 9066-9071. doi: 10.1073/pnas.1219451110

Everard, A., Lazarevic, V., Derrien, M., Girard, M., Muccioli, G. M., Neyrinck, A. M., et al. (2011). Responses of gut microbiota and glucose and lipid metabolism to prebiotics in genetic obese and diet-induced leptinresistant mice. Diabetes Metab. Res. Rev. 60, 2775-2786. doi: 10.2337/db110227

Everard, A., Lazarevic, V., Gaia, N., Johansson, M., Stahlman, M., Backhed, F., et al. (2014). Microbiome of prebiotic-treated mice reveals novel targets involved in host response during obesity. ISME J. 8, 2116-2130. doi: 10.1038/ismej. 2014.45

Forslund, K., Hildebrand, F., Nielsen, T., Falony, G., Le Chatelier, E., Sunagawa, S., et al. (2015). Disentangling type 2 diabetes and metformin treatment signatures in the human gut microbiota. Nature 528, 262-266. doi: 10.1038/nature 15766

GBD 2015 Obesity Collaborators, Afshin, A., Forouzanfar, M. H., Reitsma, M. B., Sur, P., Estep, K., et al. (2017). Health effects of overweight and obesity in 195 countries over 25 Years. N. Engl. J. Med. 377, 13-27. doi: 10.1056/ NEJMoa 1614362

Gibson, G. R., Hutkins, R., Sanders, M. E., Prescott, S. L., Reimer, R. A., Salminen, S. J., et al. (2017). Expert consensus document: the International Scientific Association for Probiotics and Prebiotics (ISAPP) consensus statement on the definition and scope of prebiotics. Nat. Rev. Gastroenterol. Hepatol. 14, 510-526. doi: $10.1038 /$ nrgastro. 2017.75

Gibson, G. R., and Roberfroid, M. B. (1995). Dietary modulation of the human colonic microbiota: introducing the concept of prebiotics. J. Nutr. 125, 1401-1412.

Gomez-Gallego, C., Pohl, S., Salminen, S., De Vos, W. M., and Kneifel, W. (2016). Akkermansia muciniphila: a novel functional microbe with probiotic properties. Benef. Microbes 7, 571-584. doi: 10.3920/BM2016.0009

Grander, C., Adolph, T. E., Wieser, V., Lowe, P., Wrzosek, L., Gyongyosi, B., et al. (2017). Recovery of ethanol-induced Akkermansia muciniphila depletion ameliorates alcoholic liver disease. Gut doi: 10.1136/gutjnl-2016-313432 [Epub ahead of print].

Greer, R. L., Dong, X., Moraes, A. C., Zielke, R. A., Fernandes, G. R., Peremyslova, E., et al. (2016). Akkermansia muciniphila mediates negative effects of IFN $\gamma$ on glucose metabolism. Nat. Commun. 7:13329. doi: $10.1038 /$ ncomms13329

Hartstra, A. V., Bouter, K. E., Backhed, F., and Nieuwdorp, M. (2015). Insights into the role of the microbiome in obesity and type 2 diabetes. Diabetes Care 38, 159-165. doi: $10.2337 / \mathrm{dc} 14-0769$

Hill, C., Guarner, F., Reid, G., Gibson, G. R., Merenstein, D. J., Pot, B., et al. (2014). Expert consensus document: the international scientific association for probiotics and prebiotics consensus statement on the scope and appropriate use of the term probiotic. Nat. Rev. Gastroenterol. Hepatol. 11, 506-514. doi: $10.1038 /$ nrgastro.2014.66

Jeurink, P. V., van Bergenhenegouwen, J., Jimenez, E., Knippels, L. M., Fernandez, L., Garssen, J., et al. (2013). Human milk: a source of more life than we imagine. Benef. Microbes 4, 17-30. doi: 10.3920/BM2012. 0040

Korpela, K., Flint, H. J., Johnstone, A. M., Lappi, J., Poutanen, K., Dewulf, E., et al. (2014). Gut microbiota signatures predict host and microbiota responses to dietary interventions in obese individuals. PLOS ONE 9:e90702. doi: 10.1371/ journal.pone. 0090702

Leal-Diaz, A. M., Noriega, L. G., Torre-Villalvazo, I., Torres, N., AlemanEscondrillas, G., Lopez-Romero, P., et al. (2016). Aguamiel concentrate from Agave salmiana and its extracted saponins attenuated obesity and hepatic steatosis and increased Akkermansia muciniphila in C57BL6 mice. Sci. Rep. 6:34242. doi: 10.1038/srep34242

Li, J., Lin, S., Vanhoutte, P. M., Woo, C. W., and Xu, A. (2016). Akkermansia muciniphila protects against atherosclerosis by preventing metabolic endotoxemia-induced inflammation in apoe-/- mice. Circulation 133, 2434-2446. doi: 10.1161/CIRCULATIONAHA.115.019645

Li, J., Zhao, F., Wang, Y., Chen, J., Tao, J., Tian, G., et al. (2017). Gut microbiota dysbiosis contributes to the development of hypertension. Microbiome 5:14. doi: $10.1186 / s 40168-016-0222-\mathrm{x}$

Liu, T. W., Cephas, K. D., Holscher, H. D., Kerr, K. R., Mangian, H. F., Tappenden, K. A., et al. (2016). Nondigestible fructans alter gastrointestinal barrier function, gene expression, histomorphology, and the microbiota profiles of diet-induced obese C57BL/6J mice. J. Nutr. 146, 949-956. doi: 10.3945/jn.115.22 7504

Marchesi, J. R., Adams, D. H., Fava, F., Hermes, G. D., Hirschfield, G. M., Hold, G., et al. (2016). The gut microbiota and host health: a new clinical frontier. Gut 65, 330-339. doi: 10.1136/gutjnl-2015-309990

Maurer, A. D., Eller, L. K., Hallam, M. C., Taylor, K., and Reimer, R. A. (2010). Consumption of diets high in prebiotic fiber or protein during growth influences the response to a high fat and sucrose diet in adulthood in rats. Nutr.Metab. (Lond) 7:77. doi: 10.1186/1743-7075-7-77

Ojo, B., El-Rassi, G. D., Payton, M. E., Perkins-Veazie, P., Clarke, S., Smith, B. J., et al. (2016). Mango supplementation modulates gut microbial dysbiosis and short-chain fatty acid production independent of body weight reduction in C57BL/6 mice fed a high-fat diet. J. Nutr. 146, 1483-1491. doi: 10.3945/jn.115. 226688

Ottman, N., Huuskonen, L., Reunanen, J., Boeren, S., Klievink, J., Smidt, H., et al. (2016). Characterization of outer membrane proteome of Akkermansia muciniphila reveals sets of novel proteins exposed to the human intestine. Front. Microbiol. 7:1157. doi: 10.3389/fmicb.2016.01157

Ottman, N., Reunanen, J., Meijerink, M., Pietila, T. E., Kainulainen, V., Klievink, J., et al. (2017). Pili-like proteins of Akkermansia muciniphila modulate host immune responses and gut barrier function. PLOS ONE 12:e0173004. doi: 10.1371/journal.pone.0173004

Ouwehand, A. C., Derrien, M., de Vos, W., Tiihonen, K., and Rautonen, N. (2005). Prebiotics and other microbial substrates for gut functionality. Curr. Opin. Biotechnol. 16, 212-217. doi: 10.1016/j.copbio.2005. 01.007

Ouwerkerk, J. P., van der Ark, K. C., Davids, M., Claassens, N. J., Robert Finestra, T., de Vos, W. M., et al. (2016). Adaptation of Akkermansia muciniphila to the oxic-anoxic interface of the mucus layer. Appl. Environ. Microbiol. doi: 10.1128/AEM.01641-16 [Epub ahead of print].

Pachikian, B. D., Essaghir, A., Demoulin, J. B., Catry, E., Neyrinck, A. M., Dewulf, E. M., et al. (2012). Prebiotic approach alleviates hepatic steatosis: implication of fatty acid oxidative and cholesterol synthesis pathways. Mol. Nutr. Food Res. 57, 347-359. doi: 10.1002/mnfr.201200364

Plovier, H., Everard, A., Druart, C., Depommier, C., Van Hul, M., Geurts, L., et al. (2017). A purified membrane protein from Akkermansia muciniphila or the pasteurized bacterium improves metabolism in obese and diabetic mice. Nat. Med. 23, 107-113. doi: 10.1038/nm.4236

Png, C. W., Linden, S. K., Gilshenan, K. S., Zoetendal, E. G., McSweeney, C. S., Sly, L. I., et al. (2010). Mucolytic bacteria with increased prevalence in IBD mucosa augment in vitro utilization of mucin by other bacteria. Am. J. Gastroenterol. 105, 2420-2428. doi: 10.1038/ajg.2010.281

Reid, D. T., Eller, L. K., Nettleton, J. E., and Reimer, R. A. (2016). Postnatal prebiotic fibre intake mitigates some detrimental metabolic outcomes of early overnutrition in rats. Eur. J. Nutr. 55, 2399-2409. doi: 10.1007/s00394-0151047-2

Roberfroid, M., Gibson, G. R., Hoyles, L., McCartney, A. L., Rastall, R., Rowland, I., et al. (2010). Prebiotic effects: metabolic and health benefits. Br. J. Nutr. 104, S1-S63. doi: 10.1017/S0007114510003363

Salonen, A., Lahti, L., Salojarvi, J., Holtrop, G., Korpela, K., Duncan, S. H., et al. (2014). Impact of diet and individual variation on intestinal microbiota composition and fermentation products in obese men. ISME J. 8, 2218-2230. doi: 10.1038 /ismej.2014.63

Schneeberger, M., Everard, A., Gomez-Valades, A. G., Matamoros, S., Ramirez, S., Delzenne, N. M., et al. (2015). Akkermansia muciniphila inversely correlates with the onset of inflammation, altered adipose tissue metabolism and metabolic disorders during obesity in mice. Sci. Rep. 5:16643. doi: 10.1038/ srep16643

Seregin, S. S., Golovchenko, N., Schaf, B., Chen, J., Pudlo, N. A., Mitchell, J., et al. (2017). NLRP6 protects Il10-/- mice from colitis by limiting colonization of Akkermansia muciniphila. Cell Rep. 19, 733-745. doi: 10.1016/j.celrep.2017. 03.080

Shen, J., Tong, X., Sud, N., Khound, R., Song, Y., Maldonado-Gomez, M. X., et al. (2016). Low-density lipoprotein receptor signaling mediates the triglyceride-lowering action of Akkermansia muciniphila in genetic-induced hyperlipidemia. Arterioscler. Thromb. Vasc. Biol. 36, 1448-1456. doi: 10.1161/ ATVBAHA.116.307597 
Shetty, S. A., Hugenholtz, F., Lahti, L., Smidt, H., and de Vos, W. M. (2017). Intestinal microbiome landscaping: insight in community assemblage and implications for microbial modulation strategies. FEMS Microbiol. Rev. 41, 182-199. doi: 10.1093/femsre/fuw045

Shin, N. R., Lee, J. C., Lee, H. Y., Kim, M. S., Whon, T. W., Lee, M. S., et al. (2014). An increase in the Akkermansia spp. population induced by metformin treatment improves glucose homeostasis in diet-induced obese mice. Gut 63, 727-735. doi: 10.1136/gutjnl-2012-303839

Singh, D. P., Singh, J., Boparai, R. K., Zhu, J., Mantri, S., Khare, P., et al. (2017). Isomalto-oligosaccharides, a prebiotic, functionally augment green tea effects against high fat diet-induced metabolic alterations via preventing gut dysbacteriosis in mice. Pharmacol. Res. 123, 103-113. doi: 10.1016/j.phrs.2017. 06.015

Song, H., Chu, Q., Yan, F., Yang, Y., Han, W., and Zheng, X. (2016). Red pitaya betacyanins protects from diet-induced obesity, liver steatosis and insulin resistance in association with modulation of gut microbiota in mice. J. Gastroenterol. Hepatol. 31, 1462-1469. doi: 10.1111/jgh.13278

Thaiss, C. A., Levy, M., Korem, T., Dohnalova, L., Shapiro, H., Jaitin, D. A., et al. (2016). Microbiota diurnal rhythmicity programs host transcriptome oscillations. Cell 167, 1495-1510.e12. doi: 10.1016/j.cell.2016. 11.003

Ward, T. L., Hosid, S., Ioshikhes, I., and Altosaar, I. (2013). Human milk metagenome: a functional capacity analysis. BMC Microbiol. 13:116. doi: 10.1186/1471-2180-13-116

Wen, L., and Duffy, A. (2017). Factors influencing the gut microbiota, inflammation, and type 2 diabetes. J. Nutr. 147, 1468S-1475S. doi: 10.3945/jn. 116.240754
Yassour, M., Lim, M. Y., Yun, H. S., Tickle, T. L., Sung, J., Song, Y. M., et al. (2016). Sub-clinical detection of gut microbial biomarkers of obesity and type 2 diabetes. Genome Med. 8:17. doi: 10.1186/s13073-016-0271-6

Zeevi, D., Korem, T., Zmora, N., Israeli, D., Rothschild, D., Weinberger, A., et al. (2015). Personalized nutrition by prediction of glycemic responses. Cell 163, 1079-1094. doi: 10.1016/j.cell.2015.11.001

Zhang, X., Shen, D., Fang, Z., Jie, Z., Qiu, X., Zhang, C., et al. (2013). Human gut microbiota changes reveal the progression of glucose intolerance. PLOS ONE 8:e71108. doi: 10.1371/journal.pone.0071108

Zhu, L., Qin, S., Zhai, S., Gao, Y., and Li, L. (2017). Inulin with different degrees of polymerization modulates composition of intestinal microbiota in mice. FEMS Microbiol. Lett. 364:fnx075. doi: 10.1093/femsle/fnx075

Conflict of Interest Statement: PC and WdV are inventors on patent applications dealing with the use of $A$. muciniphila and its components in the treatment of obesity and related disorders.

The authors declare that the research was conducted in the absence of any commercial or financial relationships that could be construed as a potential conflict of interest.

Copyright (c) 2017 Cani and de Vos. This is an open-access article distributed under the terms of the Creative Commons Attribution License (CC BY). The use, distribution or reproduction in other forums is permitted, provided the original author(s) or licensor are credited and that the original publication in this journal is cited, in accordance with accepted academic practice. No use, distribution or reproduction is permitted which does not comply with these terms. 